Bazyli DEGÓRSKI O.S.P.P.E.

(Roma, Pontificia Università San Tommaso d'Aquino)

\title{
I PROTOPLASTI PALEOCRISTIANI DELL'ORDINE DEI MONACI DI SAN PAOLO PRIMO EREMITA SECONDO L'OPERA PAULINA EREMUS DI PADRE BARTOLOMEO BOLESLAWSKI
}

L'articolo prenderà in considerazione la seguente opera manoscritta di padre Bartolomeo Bolesławski: Paulina Eremus seu co[m]mentarius hystoricus in quo Ordinis Fratrum Eremitarum Sancti Pauli Primi Eremitae primordia, progressus, fundationes, privilegia, viri illustres referuntur, datata all'anno $1630^{1}$. È un codice manoscritto con 263 pagine di carta. Questo manoscritto viene conservato nell'Archivio di Jasna Góra ${ }^{2}$.

1. Caratteristica generale degli eremiti paleocristiani. Padre Bolesławski presenta gli eremiti conformemente alla conoscenza e alla mentalità del suo tempo. Infatti, fornisce soltanto ciò che ha trovato nelle fonti in suo possesso ${ }^{3}$. Secondo Bolesławski, la vita eremitica nacque sotto l'influsso della grazia di Dio. Inoltre, lo stesso Dio si prendeva miracolosamente cura degli anacoreti, liberandoli dalle prigioni, fornendo loro cibo, punendo quelli che non erano loro propizi, tramutando lo sterile deserto in terra fertile e, se necessario, attestando miracolosamente la loro innocenza ${ }^{4}$.

L'eremita andava nel deserto per incontrare personalmente Dio. Praticava una vita severa e penitenziale: alcuni passavano la vita su un'alta colonna, altri vivevano in luoghi impervi, sulle cime dei monti, in isole disabitate e in caverne, abitavano in strettissime celle sproporzionatamente più piccole rispetto al proprio stesso corpo, non lasciandole, spesso, per molti anni. A volte, stavano

${ }^{1}$ D'ora in poi, abbrevieremo quest'opera con la sigla PE.

2 La segnatura: 752.

3 Tra queste fonti un posto peculiare occupa l'opera che lo stesso Bolesławski chiama: Vetus chronicon manuscriptum Ordinis nostri, e che attualmente è nota come l'opera del Monaco Paolino, padre Gregorio Gyöngyösi: Vitae Fratrum Ordinis Heremitarum S. Pauli Primi Heremitae. Un'altra fonte è il Fragmen Panis Corvi Protoeremitici seu Reliquiae Annalium [...] Ordinis Sancti Pauli Primi Eremitae [...], Viennae 1663, di cui l'autore è il Monaco Paolino padre Andrea Eggerer. A proposito di queste fonti dell'opera di padre Bolesławski, cfr. B. Degórski, Powstanie Zakonu Paulinów w świetle «Paulina Eremus» O. Bartłomieja Bolesławskiego, ,Studia Claromontana" 3 (1982) 257-258.

${ }^{4}$ Cfr. PE pp. $24-25,28,39,42,35,57,88,93,112,115$. 
in grotte da loro scavate o, addirittura, sotto il cielo aperto. Gli eremiti si cibavano, di solito, di frutta fresca e di erbe, non bevevano il vino, non usavano l'olio o altro di gustoso e per lunghi periodi praticavano l'inedia, portavano capelli lunghi, unghie e barbe incolte, usavano il cilicio e praticavano molte penitenze corporali assai severe ${ }^{5}$.

Essi volevano rimanere soltanto alla presenza di Dio. Egli era per loro l'Altissimo, l'Immutabile, l'Incomprensibile, l'Assoluto ${ }^{6}$. La preghiera dell'eremita era semplice e spontanea, composta prevalentemente della recita dei Salmi o della contemplazione del Signore nel silenzio. In tali condizioni, il solitario poteva rimanere per l'intera giornata ${ }^{7}$. L'eremita è anche un figlio fedele della Chiesa. Padre Bolesławski presenta, ad esempio, l'eremita Giovanni il Persiano, vissuto tra il VI e il VII secolo, che dal suo eremo si rec pellegrino a Roma ${ }^{8}$. Questi asceti, inoltre, sigillarono spesso la loro unione a Cristo con lo spargimento di sangue, in quanto difesero con forza la fede anche lottando, per esempio, contro gli errori di Ario, Nestorio o l'iconoclasmo $^{9}$. Ciò nonostante, a volte, anch'essi furono influenzati da dottrine eretiche. Secondo padre Bolesławski, però, non si trattò di casi numerosi. Il nostro Autore, infatti, expressis verbis, ne elenca soltanto cinque, vissuti in Egitto tra il IV e il V secolo: Ammonio, Eusebio, Eutimio, Evagrio e Isidoro. Tutti costoro condivisero il pensiero di Origene ${ }^{10}$.

Altra forma di qualche cenno di debolezza nei primissimi eremiti furono le tentazioni di abbandono dell'eremo. Tuttavia, essi rimanevano per lo più fermi nel proprio rigido proposito e tra loro spesso si trovavano pure malfattori, ladri e altri uomini depravati che, convertiti, in tal modo cercavano di espiare i peccati commessi ${ }^{11}$.

Ciascun asceta si rendeva conto di partecipare anche alla missione della Chiesa. Spesso accadeva che qualcuno diventasse vescovo, anche se non mancavano quelli che schivavano questa dignità. Bolesławski fornisce qui, seguendo il Martyrologium Romanum, l'esempio di san Nilamone, vissuto in Egitto, il quale, forzato a diventare vescovo, rese l'anima a Dio. Lo spirito missionario degli eremiti si esprimeva anche con il fatto che, non di rado, essi abbandonavano l'eremo per qualche azione missionaria ${ }^{12}$.

5 Cfr. PE pp. 34-130.

${ }^{6}$ Cfr. PE p. 35.

7 Cfr. PE pp. 35, 48, 71 .

8 A proposito di questo pellegrinaggio di Giovanni il Persiano, cfr. Iohannes Moschus, Pratum spirituale 151, PG 87, 3016-3017

${ }^{9}$ Cfr, PE pp. 28, 32-34, 44, 46, 54, 58, 64, 70, 78, 85, 89, 92-96, 98, 100-104, 119-120.

${ }^{10}$ Cfr. PE pp. 38-39: ,[...] Ammonius et Eusebius, Eutymius et Evagrius, Isidorusq[ue] [...], qui licet vitae et solitudinis amore floruerunt, Origenis tamen macula tincti sunt [...]".

11 Cfr. PE pp. 37-39, 78, 113.

12 Cfr. Degórski, Powstanie Zakonu Paulinów, p. 261; idem, Első Remete Szent Pál szerzetesrendjének története Bartolomeo Bolesławski Paulina eremus címümúve alapján, in: Decus soli- 
A volte però, la situazione era diametralmente opposta: molti sacerdoti e vescovi abbandonavano l'apostolato attivo e si facevano eremiti. Altri, invece, sceglievano una forma intermedia: per qualche periodo lasciavano il deserto per intraprendere l'apostolato e poi ritornare, infine, all'eremo ${ }^{13}$. A causa della loro santità, fedeli e persino governanti li stimavano molto e spesso cercavano presso di loro lumi e consigli ${ }^{14}$. Ad esempio, vale la pena di citare qui l'eremita Giovanni il Persiano il quale, durante il pellegrinaggio a Roma, venne a sapere che doveva passare lì dove egli si trovava, lo stesso papa Gregorio Magno. Giovanni voleva rendere omaggio a Gregorio, ma il papa per primo cadde di fronte a lui per terra, salutò umilmente Giovanni e gli diede tre monete, ordinando poi che gli fosse dato tutto ciò che gli occorreva ${ }^{15}$.

Gli eremiti che presenta padre Bolesławski posseggono il dono della profezia, sono operatori di miracoli, anzi: persino gli oggetti appartenenti a loro o, in qualche modo, con loro collegati, compiono miracoli, come avviene anche con una semplice invocazione dei loro nomi. La loro santità e la forza taumaturgica e miracolosa che si emana da loro si rivelano pure nel fatto che le bestie feroci, per esempio, a loro ubbidiscono e si sottomettono ${ }^{16}$.

Padre Bolesławski si occupò degli eremiti veri e propri e non trattò, perciò, dei cenobiti, in quanto, pare, pensava che ciò esulasse dalla problematica della Paulina Eremus. Tuttavia, egli non poté tralasciare completamente di accennare ai cenobiti, perché spesso la loro vita fu collegata con quella degli eremiti. Per tale ragione, i cenobiti vengono qui soltanto appena menzionati. Padre Bolesławski si occupa comunque direttamente e abbastanza a lungo unicamente di san Pacomio, l'autore della vita cenobitica e discepolo dell'asceta Palemone, e dei discepoli di san Pacomio: Teodoro, Pafnuzio ed Elia, benché li annoveri tutti quali figli spirituali di san Paolo il Primo Eremita $^{17}$.

2. Periodi e diffusione dell'eremitismo paleocristiano. Esponendo su diversi eremiti paleocristiani, padre Bartolomeo li presenta in ordine cronologico. Con Baronio considera l'anno 343 la data di morte di san Paolo il Primo Eremita in base alla quale raggruppa gli eremiti ${ }^{18}$. E così, nel primo centenario dopo la morte di san Paolo di Tebe (343-443), secondo Bolesławski lo stile di

tudinis. Pálos évszázadok [= Múvelődéstörténeti Múhely. Rendtörténeti konferenciák, 4/1], ed. G. Sarbak, Budapest 2007, 109.

13 Cfr. PE pp. 55, 59, 74, 81-82, 92, 99-100, 105, 109, 112, 114.

${ }^{14}$ Cfr. PE pp. 37, 43, 55-56, 77, 81, 88, 93-94, 102, 112, 118, 121.

${ }^{15}$ Cfr. PE pp. 89-90. La fonte dalla quale Bolesławski attinse queste notizie è la seguente: Iohannes Moschus, Pratum spirituale 151, PG 87, 3016-3017.

${ }^{16}$ Cfr. Degórski, Powstanie Zakonu Paulinów, p. 262; idem, Elsö Remete Szent Pál, p. 109.

17 Cfr. PE p. 33.

18 Cfr. Degórski, Powstanie Zakonu Paulinów, p. 262; idem, Elsó Remete Szent Pál, p. 110 
vita del grande Tebano era divenuto ormai noto in Egitto, in Siria, in Palestina, in Italia e in Gallia. Dall'Egitto alla Siria e alla Palestina questa forma ascetica fu trapiantata da uno dei discepoli di sant'Antonio: sant'Ilarione; alla Grecia, invece, giunse grazie a san Basilio Magno, san Gregorio di Nazianzo e san Giovanni Crisostomo ${ }^{19}$.

Presentando, quindi, gli eremiti vissuti nel secondo periodo dopo la morte di san Paolo di Tebe (443-643), Bolesławski tratta soltanto di quelli dell'Egitto, della Siria, della Grecia e della Palestina ${ }^{20}$. Bisogna dire che la sua posizione è corretta. Infatti, si sa che il movimento eremitico-monastico giunse in Occidente proprio nel IV secolo, allorché arrivò in Italia, e in quel periodo era già presente anche nelle isole limitrofe alla penisola italiana e in Gallia. Nel secolo $\mathrm{V}$ gli eremiti sono presenti in Provenza e, dal V all'VIII secolo, in Irlanda, in Scozia, in Portogallo e in Spagna e persino nel centro dell'Europa ${ }^{21}$, anche se, a dir il vero, l'eremitismo giunse in quelle regioni, specialmente in Ungheria, soltanto nell'età carolingia ${ }^{22}$.

3. Gli eremiti e san Paolo il Primo Eremita. Volendo dimostrare la stretta unione dei primissimi eremiti con san Paolo di Tebe, Bolesławski presenta dettagliatamente la storia dell'eremitismo e del monachesimo paleocristiano. Egli tratta la storia dell'eremitismo a partire proprio da san Paolo il Primo Eremita che, durante la persecuzione di Decio, fuggì nel deserto della Tebaide dove visse in solitudine e morì all'età di 113 anni $^{23}$.

Padre Bartolomeo non presenta dettagliatamente la vita di san Paolo, ma la cita abbastanza chiaramente. Non lo fa neppure con un unico riferimento, ma in diversi punti della Paulina Eremus, in cui presenta la persona del primo eremita di Tebe come principe degli eremiti e autore di questo genere di vita ascetica. Bisogna precisare che, oltre la data della nascita di san Paolo, il 230, e il luogo, notizie tutte attinte da Baronio, Bolesławski non fornisce alcun altro particolare della sua vita.

${ }^{19}$ Cfr. PE pp. 28, 30-36, 47; vedi anche PE pp. 59-64. Così afferma anche un autore anonimo che firma la sua opera con le lettere „L.N.”; cfr. L.N., Radix et origo Eremi - Coenobiticae Religionis S. Pauli Primi Eremitae, Romae 1683, 3. Cfr anche Degórski, Powstanie Zakonu Paulinów, p. 262; idem, Elsó Remete Szent Pál, p. 110.

${ }^{20}$ Cfr. Degórski, Powstanie Zakonu Paulinów, p. 262; idem, Elsó Remete Szent Pál, p. 110.

21 Cfr. Ph. Rouillard, Eremitismo, DIP III 1230.

22 Cfr. J. Gajsler, Dzieje Węgier w zarysie, I, Warszawa 1898, 43; G. Deer, La formazione del Regno d'Ungheria, Budapest 1943, 112; J. Kłoczowski, Chrześcijaństwo czasów karolińskich, „Znak” 14 (1962) n. 91, p. 3.

23 Non conosciamo precisamente la data della morte di san Paolo di Tebe. B. Bolesławski (PE p. 9) e A. Eggerer (Fragmen Panis Corvi Protoeremitici, p. 31), basandosi sul calcolo di Cesare Baronio, la stabiliscono per l'anno 343. A proposito della vita di san Paolo di Tebe, cfr. Hieronymus, Vita S. Pauli Primi Eremitae, in: Edizione critica della «Vita Sancti Pauli Primi Eremitae» di Girolamo, ed. R. [= B.] Degórski, Roma 1987, Institutum Patristicum «Augustinianum». 
Per annoverare tutti gli eremiti nella cerchia dei seguaci di san Paolo di Tebe $^{24}$, Bolesławski si basa sulla seguente affermazione di san Girolamo, tratta dalla lettera a Eustochio:

„Passo [...] a parlare della terza categoria, coloro che son chiamati anacoreti: essi abbandonano i cenobi, e vanno nel deserto portando con sé unicamente pane e sale. Il fondatore di questo genere di vita è Paolo, ma chi lo rese celebre è Antonio, e se vogliamo risalire alle origini, il primo esempio è stato Giovanni Battista"25.

In questo modo, Bolesławski include nel numero degli imitatori del primo eremita tutti quanti i solitari a lui noti e vissuti dopo il grande solitario della Tebaide. Inoltre, per quanto possibile, cerca di fornire dei particolari che possono giustificare l'unione di un eremita a tutti noto con san Paolo di Tebe. E così, cerca di presentare una serie di emuli paolini e lo fa a partire dal più grande propagatore della fama di quel primo solitario, cioè da sant'Antonio abate. Lo presenta basandosi sulla Vita S. Pauli di san Girolamo e afferma che sant'Antonio fu il maggior diffusore della vita solitaria di san Paolo di Tebe. Commentando, ad esempio, l'incontro di sant'Antonio con san Paolo, Bolesławski sottolinea l'ammirazione di sant'Antonio per san Paolo. Infatti, egli che si considerava il primo e più severo asceta, veniva, in realtà, di gran lunga superato da san Paolo. Da questo elemento Bolesławski constata che, grazie all'aiuto di san Paolo, sant'Antonio divenne il secondo esponente e seguace dell'eremitismo paolino ${ }^{26}$. L'autore cita anche le parole che san Paolo rivolse a sant'Antonio: „expedit Fratribus ut tuo instituantur exemplo"27. Commentando questa frase, padre Bolesławski afferma che sant'Antonio voleva così dirigere i suoi solitari, affinché l'esempio della vita di san Paolo avesse potuto diventare l'occasione per diffondere questo genere di ascesi. Nota, inoltre, che sant'Antonio, dopo la morte di san Paolo, divenne veramente il maggiore divulgatore in Egitto della sua vita eremitica: lo prese come principe e norma di vita, organizzò la propria cella similmente a quella di san Paolo e, infine, si sa che indossava la tunica di san

${ }^{24}$ Per quanto riguarda la presenza di san Paolo Primo Eremita nei testi patristici, cfr. B. Degórski, Święty Pawet Pierwszy Pustelnik w świetle tekstów patrystycznych, ,Studia Claromontana" 6 (1985) 110-144.

25 Hieronymus, Epistula 22 [De virginitate servanda] 36, CSEL 54, 200: , [...] ad tertium genus veniam, quos anachoretas vocant et qui de coenobiis exeuntes excepto pane et sale amplius ad deserta nil perferunt. Huius vitae auctor Paulus, inlustrator Antonius et, ut ad superiora conscendam, princeps Iohannes baptista fuit". Bolesławski si appella spesso a questo brano geronimiano, cfr. ad esempio: PE pp. 7, 9, 11, 22, 32, 49 ecc.

${ }^{26}$ Cfr. PE pp. 10-11. Bolesławski lo fa usando le seguenti parole: „Ex his liquido constat, quod S. Antonius Frater et conservus D. Pauli existens [...] Succedaneus Author extiterit" (PE p. 11).

${ }^{27}$ Il testo critico di questa opera geronimiana suona così: cfr. Hieronymus, Vita S. Pauli Primi Eremitae 12, in: Edizione critica, pp. 143-144: ,[...] expedit fratribus, ut tuo adhuc instituantur exemplo". 
Paolo. Il nostro Autore lo afferma citando il Carmen ad Faustum di Sidonio Apollinare $^{28}$.

Temendo un'eventuale accusa sul fatto che sant'Antonio fosse piuttosto il padre dei cenobiti e non degli eremiti, padre Bolesławski, basandosi su Baronio, scrive che quest'abate dapprima fece da anacoreti cenobiti e, in seguito, sotto l'influsso di san Paolo, da cenobiti anacoreti. Quindi, può essere giustamente chiamato il principe sia degli uni che degli altri ${ }^{29}$. Cercando di appoggiare ulteriormente la stretta dipendenza degli eremiti paleocristiani da san Paolo di Tebe, Bolesławski cita un frammento preso dalla lettera di san Girolamo a san Paolino di Nola nella quale, tra l'altro, leggiamo:

„Per ogni ideale c'è un modello da imitare: i condottieri romani imitino i Camilli, i Fabrizi, i Regoli, gli Scipioni; i filosofi si specchino in Pitagora, in Socrate, in Platone, in Aristotele; i poeti cerchino di emulare Omero, Virgilio, Menandro, Terenzio; e gli storici Tucidide, Sallustio, Erodoto, Livio; gli oratori poi hanno Lisia, i Gracchi, Demostene e Cicerone. E, per tornare a noi, i vescovi e i sacerdoti si tengano davanti, come esempi, gli Apostoli e gli uomini apostolici. Partecipano del medesimo onore? Si sforzino di averne anche il merito. Quanto a noi, i modelli del nostro ideale di vita sono i Paolo, gli Antonio, i Giuliano, gli Ilarione e i Macario" 30 .

In seguito, padre Bartolomeo indica tra le persone collegate spiritualmente con san Paolo di Tebe alcune grandi figure dell'Antico Testamento, quali ad esempio Elia ed Eliseo ${ }^{31}$. Possiamo chiederci perché Bolesławski non vede la genesi dell'eremitismo nell'Antico Testamento dato che, senza dubbio, questo stile di vita in qualche modo ha le sue radici in quel tempo ${ }^{32}$ e non era sconosciuto neppure nell'età apostolica ${ }^{33}$.

${ }^{28}$ Cfr. PE p. 23: ,Nunc vocat in tunica te Antonius illa, / Quam fecit palma foliis manus alma Magistri"; Sidonius Apollinaris, Carmen 16 [ad Faustum Riensem], PL 58, 720C.

${ }^{29}$ Cfr. PE p. 52: ,[...] is exemplo suo [...] nunc ex anachoretis faceret caenobitas, nunc vice versa ex Caenobitis Anachoretas, ut tam Anachoretarum quam Caenobitarum dicendus sit princeps magnificus".

30 Hieronymus, Epistula 58, 5, CSEL 54, 533-534 (ed. e trad. it. S. Cola: San Girolamo, Le Lettere, II, Roma 1997, 133-134): „Habet unumquodque propositum principes suos: Romani duces imitentur Camillos, Fabricios, Regulos, Scipiones; philosophi proponant sibi Pythagoram, Socraten, Platonem, Aristotelen; poetae aemulentur Homerum, Vergilium, Menandrum, Terentium; historici Thucydiden, Sallustium, Herodotum, Livium; oratores Lysiam, Gracchos, Demosthenen, Tullium; et ut ad nostra ueniamus, episcopi et presbyteri habeant in exemplum apostolos et apostolicos viros, quorum honorem possidentes habere nitantur et meritum. Nos autem habemus propositi nostri principes Paulos, Antonios, Iulianos, Hilariones, Macarios [...]”.

${ }^{31}$ Cfr. PE pp. 21-22 e 51.

32 Cfr. J. Leclercq, L'érémitisme en Occident jusqu'à l'an mil, in: L'eremitismo in Occidente nei secoli XI e XII, Milano 1965, 29-30; G. Pelliccia - G. Rocca, Eremitismo, DIP III 1225; J. Gribomont, Eremo, DIP III 1260-1262.

33 Cfr. ad esempio Gribomont, Eremo, p. 1262. 
La risposta a tale questione si trova nella Paulina Eremus, dove Bolesławski considera l'eremo del profeta Elia una forma di vita cenobitica e non eremitica e, conseguentemente, cerca di spiegare questo frammento della lettera geronimiana nel quale il Dalmata indica proprio Elia ed Eliseo quali predecessori spirituali di san Paolo di Tebe. Porta qui come esempio sant'Antonio da Padova, che dapprima era canonico regolare e poi divenne francescano. Per questa ragione, ambo gli ordini lo venerano come proprio membro. Similmente, san Girolamo passò dall'eremo cenobitico di Elia alla solitudine rigorosa di san Paolo di Tebe, il che può essere dimostrato dalla sua lettera al monaco Rustico, nella quale, tra l'altro, scrive:

„Condanno per caso la vita eremitica? Nient'affatto; anzi più volte ne ho tessuto l'elogio. Ma vorrei che dalla palestra dei monasteri uscissero soldati che non hanno paura della gavetta, soldati che abbiano dato prova per lungo tempo di saper condurre questo genere di vita [...]"34.

In seguito, Bolesławski afferma che san Girolamo conosceva la dottrina di sant'Agostino d'Ippona, il quale nel capitolo 23 del De operibus monachorum riteneva più santi coloro che vivevano da eremiti. La stessa opinione, secondo Bolesławski, condivise san Tommaso d'Aquino ${ }^{35}$. Inoltre, per quanto riguarda san Girolamo, il fatto che egli visse anche nel monastero a Betlemme, non può negare che egli stesso fosse stato anche eremita. La vita claustrale, infatti, era una delle forme più diffuse di vita consacrata a Dio e, praticandola, egli viveva santamente come fosse eremita: era ritenuto particolarmente santo e seguace sia dell'eremo cenobitico di Elia che di quello rigoroso e perfetto di san Paolo di Tebe. Lo stesso stile di vita condivisero anche san Medardo e san Romualdo, il quale era vissuto dapprima come benedettino e, in seguito, cominciò ad imitare san Paolo il Primo Eremita, divenendo poi il padre dei camaldolesi. Uguale esperienza fece sant'Ilarione (†371 ca.), che trasferì lo stile eremitico di vita dall'Egitto alla Siria e alla Palestina. Un simile comportamento si incontra, inoltre, in san Macario, sant'Amata, Paolo il Semplice, Cronio, Origene e Pitirione. I discepoli di Ilarione, infine, vengono ricordati nelle figure di Epifanio, il futuro vescovo di Salamina, e di Esichio.

E qui termina quest'ininterrotta serie di seguaci e di figli spirituali di san Paolo di Tebe. Ciò nonostante, Bolesławski segnala ancora diversi altri eremiti e indica dei collegamenti tra di loro. Per esempio - basandosi sulla lettera di san Girolamo a Paolino, sulla testimonianza del Cardinale Baronio nonché sull'omelia 22 sulla Lettera agli Efesini di san Giovanni Crisostomo - presenta

${ }^{34}$ Cfr. PE p. 53; Hieronymus, Epistula 125, 9, CSEL 56, 128, Cola p. 278: „Quid igitur? Solitariam vitam reprehendimus? Minime, quippe quam saepe laudavimus sed de ludo monasteriorum huiusce modi volumus egredi milites, quos rudimenta non terreant, qui specimen conversationis suae multo tempore dederint [...]".

${ }^{35}$ Cfr. PE pp. 53-54; vedi anche Thomas Aquinas, Summa Theologiae II-II, 188 ad 8. 
l'anacoreta Giuliano il Siro quale erede della spiritualità di san Paolo di Tebe e maestro degli eremiti Giacomo e Acacio, un futuro vescovo, quest'ultimo, che praticò ancora lo stesso stile eremitico di vita $(\dagger 436)^{36}$.

Quando Bolesławski non riesce ad indicare precisamente la successione degli asceti solitari a partire da san Paolo il Primo Eremita, presenta allora delle piccole, a volte insignificanti, somiglianze tra gli eremiti e san Paolo. E così, annovera san Simone lo Stilita tra i seguaci di Paolo soltanto perché, tra l'altro, come Paolo, ,separato dallo strepito del mondo, in ginocchio, concluse la corsa della vita". Ricordati sono anche i cristiani africani perseguitati dai Vandali. Infatti, citando l'opera sulle persecuzioni vandaliche nell'Africa latina, composte da Vittore di Vita, scrive che molti cristiani dell'Africa settentrionale, temendo le violenze dei Vandali ariani, si nascondevano in luoghi solitari e lì, privi di ogni aiuto, morivano. In queste condizioni essi rinvenirono il sacerdote Cresconio, che già era in decomposizione. Basandosi su tali esempi, Bolesławski conclude:

„Trovando questi illustrissimi uomini come emuli di Paolo non esito di annoverarli nel numero dei successori di san Paolo il Primo Eremita" ${ }^{37}$.

Non finisce qui, però, la sua argomentazione. Infatti, a questo punto, parla di sant'Onofrio, il maestro di san Pafnuzio, sottolineando che Pafnuzio, così come all'epoca sant'Antonio abate aveva trovato san Paolo nel deserto, anch'egli aveva incontrato sant'Onofrio. Presentando la vita di san Palemone, fornita in base alle Vitae Patrum di Giovanni Mosco, cita una leggenda secondo la quale, durante le fredde notti invernali, veniva riscaldato da un leone. In tal modo, l'asceta era simile a san Paolo di Tebe, al quale gli animali prestavano servizio. A proposito di Anonimo l'Anacoreta scrive che visse per quarantacinque anni in una stretta torretta e, in tal modo, divenne un vero imitatore e seguace dell'eremitismo iniziato da san Paolo il Primo Eremita.

Di simili altri eremiti potremmo indicare ancora assai di più. Infatti, Bolesławski menziona degli eremiti dei quali non conosceva affatto la vita o, addirittura, gli era sconosciuto il periodo in cui vissero. Ne fornisce l'informazione soprattutto basandosi sul Martyrologium Romanum e dedica a loro tutto il capitolo IX, intitolato: „Qui Viri gloriosi Paulinam Eremum coluerunt incertis temporibus, veritate tamen certissimi?" 38 .

Presentando diversi eremiti, padre Bartolomeo cercò anche di respingere delle eventuali accuse che egli avesse considerato eremiti coloro che non lo erano. Infatti, citando le vite degli eremiti orientali del VI secolo, tratte da Sofronio: Leone di Cappadocia, Giovanni, Eustazio di Roma e di Teodoro,

\footnotetext{
36 Cfr. PE pp. 24-26, 28 e 47.

37 PE p. 70.

38 PE pp. 111-117.
} 
cerca di spiegare il titolo „Abbas" che Sofronio attribuisce a Leone. Questa difficoltà viene spiegata da Bolesławski nel senso che detto titolo si addice anche agli eremiti. Anzi, è il titolo che veniva loro dato sia a causa della innocenza della loro vita, sia dell'età avanzata sia, infine, a causa delle pratiche penitenziali da loro esercitate ${ }^{39}$. In questo ampio catalogo di tali santi non mancano neppure i cenobiti. Bolesławski menziona, infatti, tra gli altri, i „fratelli" che vivevano nei monasteri del deserto egiziano e che nel V secolo furono uccisi dai barbari. Circa costoro conclude: „, non si può dubitare che questi martiri appartenevano alla solitudine paolina".

Bolesławski presenta pochissime eremite. Ne nomina soltanto due: Antusa e Teoctiste. Inoltre, anche se le chiama „eremite”, non le annovera tra i seguaci di san Paolo di Tebe ${ }^{40}$. Bolesławski, ovviamente, non afferma che tutti questi eremiti erano consapevoli di imitare la vita iniziata dal grande Paolo di Tebe, ma che, a causa dello stesso stile di vita, essi possono essere considerati veri paolini. Riassumendo questa presentazione degli anacoreti, l'autore scrive:

„Dal momento che Paolo divenne il principe e l'autore degli eremiti, elaborando nel deserto lo stile eremitico di vita, è logico considerare nel numero degli emuli della forma ascetica paolina tutti coloro che brillarono con un simile genere di vita e proclamarli veri eremiti paolini. Infatti, di quali eremiti egli fu l'autore, principe e maestro se non di loro? Anche perché Gregorio XIII, nella bolla data ai Paolini, palesemente insegna che il nostro ordine fu istituito da san Paolo il Primo Eremita grazie alla santità di vita e ad un eccezionale proposito. Quindi, è logico affermare che tutti gli eremiti paolini divennero veri padri e fratelli. Per questo motivo, giustamente canta ogni mese il coro dei paolini il seguente inno: «Paolo è l'autore e il maestro degli eremiti»" 41 .

4. I Monaci Paolini e san Paolo il Primo Eremita. L'autore della Paulina Eremus considera san Paolo di Tebe il primo e principale ,istitutore" dell'ordine paolino e scrive di lui che è colui che „fondò il suo ordine eremitico non con le parole o per iscritto, ma piuttosto con l'esempio e il modo della vita". Ciò non significa affatto che l'ordine paolino fosse fondato formalmente da san Paolo. Egli fu soltanto la fonte dalla quale attinsero santi eremiti. Bolesławski riassume tutto questo lungo processo, scrivendo che:

,tale istituzione iniziò a crescere da una piccola fonte, da questo san Paolo il Primo Eremita. Da lui altri santi eremiti ed anacoreti discesero come flutti che scorrono lentamente e, con il passare del tempo, consolidarono la pia emulazione. Anch'essi, infine, disseminati nei deserti e dai deserti riuniti in monasteri, vi crebbero in

\footnotetext{
${ }^{39}$ Cfr. PE pp. 86-87.

${ }^{40}$ Cfr. Degórski, Powstanie Zakonu Paulinów, p. 269.

${ }^{41}$ Ibidem, pp. 269-270.
} 
ampie ed enormi vette di virtù claustrali. E così il nostro ordine proto-eremitico, nato e piantato in Egitto e propagato dagli eremiti, finalmente, quando venne dal beneplacito divino la pienezza dei tempi, accettò lo stile di vita claustrale" ${ }^{, 42}$.

Questa non è soltanto l'opinione di Bolesławski, perché questo schema si incontra anche presso gli altri storici dell'ordine paolino ${ }^{43}$. Egli stesso, inoltre, si appella a detta comune opinione e scrive che ,il nostro ordine sempre confessa questa verità" ${ }^{44}$. Seguendo il medesimo metodo, Bolesławski parla delle opinioni di alcuni seguaci di Lutero che ,hanno osato espellere san Paolo il Primo Eremita non soltanto dal cielo, ma anche dalla natura delle cose" ${ }^{" 45}$. Ciò può negare anche l'esistenza dei Monaci Paolini, perché, ,,tolto [...] il Padre degli eremiti, si può facilmente cancellare l'ordine eremitico" ${ }^{46}$. Bolesławski, però, non si preoccupa più di tanto di questa questione ed è persuaso che l'opinione dei luterani sia già stata sufficientemente respinta dal cardinale Roberto Bellarmino nel De Monachismo ${ }^{47}$ e da Cesare Baronio negli Anna$\operatorname{les}^{48}$. Bolesławski, tuttavia, cerca di fornire almeno qualche argomento che approvi la dipendenza dei Monaci Paolini dallo stesso san Paolo di Tebe e, quindi, l'antichità dell'Ordine paolino. Con padre Gregorio Gyöngyösi afferma che l'Ordine paolino è ,proto-eremitico" e che questa verità non può essere messa in questione ${ }^{49}$.

Un altro argomento che testimonia l'antichità dei Monaci Paolini e della loro unione con l'eremo di san Paolo, sono antiche cronache, scritti autentici o, infine, i privilegi concessi all'Ordine. Tutto ciò testifica unanimemente che l'Ordine prese inizio in Egitto e può essere ritenuto il più antico di tutti gli ordini religiosi. Per approvare questo, Bolesławski scrive che i Monaci Paolini furono comunemente chiamati ,,avi” e non ,padri” o ,fratelli”,50. L'antichità dell'Ordine paolino viene indicata anche dai testi liturgici propri dell'Ordine dedicati, tra l'altro, a san Paolo, o anche dall'iconografia, che presenta l'Anacoreta di Tebe nel bianco abito monastico paolino. Senza dubbio, l'autore si rende conto che quest'argomentazione è forzata e semplicistica. Non gli è sconosciuta l'opinione del domenicano Abramo Bzowski e di Giovanni Goto-

42 PE p. 4.

${ }^{43}$ Cfr. ad esempio: Eggerer, Fragmen Panis Corvi Protoeremitici, pp. 28-50; L.N., Radix et origo Eremi, p. 3; G. Mallechich, Quadripartitum Regularium [...] de privilegiis [...] Ordinis S. Pauli Primi Eremitae [...], Viennae 1708; I. Pongracz, Triumphus Pauli, Posonii 1752, 13-14; Acta sincera S. Pauli Thebaei cognomento Primi Eremitae graeco-latina, ed. M. Fuhrmann, Neostadii 1760.

${ }^{44}$ PE p. 4.

45 Ibidem.

46 PE p. 5.

47 Nel capitolo 5 del libro VII.

48 T. 3, anno Christi 343, n 23.

49 Cfr. Degórski, Powstanie Zakonu Paulinów, p. 270.

${ }^{50}$ Cfr. ibidem, p. 271. 
fredo che respingono decisamente la diretta dipendenza del Monaci Paolini con l'Egitto eremitico paleocristiano ${ }^{51}$.

Padre Bartolomeo, subito dopo, parla della bolla di Martino V Sacrae religionis, del 28 dicembre 1417, nella quale il papa scrive che l'Ordine paolino è nato recentemente in Ungheria ${ }^{52}$. Secondo l'autore, però, ciò non vieta di affermare che i paolini sorti in Ungheria provengano anche direttamente dallo stile di vita sia del primo eremita cristiano che dei suoi più antichi seguaci. Ciò, infatti, viene suggerito dal privilegio che i Monaci Paolini ottennero dal legato pontificio, il Cardinale Gentile, nel documento Qui saecularia, del 1308, nel quale il legato afferma che questi eremiti avevano chiesto a Urbano IV il permesso di poter vivere in comunità, ma conservando il titolo di eremiti. Concludendo, perciò, Bolesławski non esita di affermare che una cosa è il fatto che questi eremiti fossero riuniti in comunità, e un'altra che la loro ininterrotta unione spirituale risalisse a quell'eremitismo che dall'Egitto attraverso la Siria e la Grecia era poi giunto in Ungheria causando la nascita dei Monaci Paolini ${ }^{53}$.

Non importa qui che san Paolo di Tebe sia stato preso come patrono da altre comunità religiose e ordini religiosi, specialmente dagli eremiti di sant'Agostino i quali lo venerano liturgicamente e godono, a causa di ciò, anche di privilegi papali propri. Infatti, il possedere san Paolo come patrono non è importante, perché, ad esempio, i Monaci Paolini venerano notevolmente san Filippo Neri, ma ciò non significa che i religiosi oratoriani ne siano gelosi. Anzi, le comunità religiose scambiano tra di loro diversi privilegi che, nel caso dei Monaci Paolini, viene praticato riguardo all'Arciconfraternita degli Angeli Custodi. Ritornando agli Agostiniani, padre Bolesławski dice che lo stesso sant'Agostino d'Ippona fu seguace di san Paolo di Tebe. Non possiamo, dunque, meravigliarci che gli Agostiniani abbiano scelto come patrono questo primo eremita. Comunque, lo storico agostiniano Nicola Krusenius ${ }^{54}$ vede gli inizi dell'eremitismo cristiano in sant'Agostino e l'inizio dell'Ordine Agostiniano scorge nei suoi discepoli sparsi nei deserti dell'Egitto i propri „padri”. Ovviamente, Bolesławski non approva quest'opinione e, assieme a san Girolamo, sostiene che l'assoluta priorità qui deve essere data esclusivamente a san Paolo di Tebe e, invece, a sant'Antonio abate il ruolo di essere propagatore dello stile di vita iniziato dal primo eremita cristiano. Per approvarlo, cita un frammento di un sermone di sant'Agostino nel quale menziona sua madre Monica, la quale aveva parlato al figlio di san Paolo di Tebe e di sant'Antonio l'abate. Ciò fece sì che sant'Agostino divenisse discepolo e

\footnotetext{
51 Cfr. ibidem, p. 271.

52 Cfr. PE p. 14.

${ }^{53}$ Cfr. ibidem.

${ }^{54}$ Cfr. PE p. 20. Bolesławski si appella qui al capitolo 18 del De monachismo di Krusenius.
} 
seguace dell'eremitismo paolino. Per tale ragione, i Monaci Paolini chiesero alla Santa Sede la Regula di sant'Agostino, in quanto proprio in essa videro un efficace strumento ed aiuto per mantenere lo spirito eremitico paolino, mentre nella persona di sant'Agostino ne poterono scorgere un concreto propagatore $^{55}$.

L'opera manoscritta Paulina Eremus di Bolesławski non è soltanto una preziosa fonte per conoscere la storia dell'Ordine dei Monaci di San Paolo Primo Eremita, ma anche uno studio per approfondire la storia dell'eremitismo paleocristiano. Con quest'esperienza l'Ordine fu sempre strettamente collegato dagli stessi primordi. La Paulina Eremus fornisce molti particolari riguardanti sia la storia dell'eremitismo che dello stesso Ordine paolino. Essa dimostra che la spiritualità dei Monaci Paolini è ancorata non solo al primo eremita cristiano, bensì a tutti gli altri eremiti dei primissimi secoli della Chiesa.

Tale importante fonte del carisma dell'Ordine paolino, più che dei suoi protagonisti, non sempre presentati o persino più profondamente conosciuti, sembra seguire quasi idealmente una sottile analogia con quella che è la tradizione apostolica. L'Autore, infatti, non tanto si preoccupa dei dati biografici e della cronologia o verità storica degli elementi che presenta, quanto piuttosto del dimostrare l'ininterrotta continuità di grazia di un dono spirituale che, seppure ha pallide figure nell'Antico Testamento, è con il Battista e san Paolo di Tebe, quasi suo emulo tra persecuzione e vita ritirata nel deserto, che si genera in seno al cristianesimo, si radica nel tessuto ecclesiale e si diffonde di secolo in secolo propagandosi come il Vangelo e con il Vangelo sino a terre lontane.

\author{
STAROCHRZEŚCIJAŃSCY PROTOPLAŚCI ZAKONU ŚW. PAWŁA \\ PIERWSZEGO PUSTELNIKA WEDEUG DZIEŁA \\ PAULINA EREMUS O. BARTEOMIEJA BOLESŁAWSKIEGO
}

(Streszczenie)

Artykuł analizuje rękopiśmienne dzieło o. Bartłomieja Bolesławskiego O.S.P.P.E.: Paulina Eremus seu co[m]mentarius hystoricus in quo Ordinis Fratrum

55 Cfr. PE p. 20. 
Eremitarum Sancti Pauli Primi Eremitae primordia, progressus, fundationes, privilegia, viri illustres referuntur, które pochodzi z 1630 roku. Manuskrypt ten jest obecnie przechowywany w Archiwum Jasnogórskim. Paulina Eremus o. Bolesławskiego jest nie tylko cennym źródłem, by poznać dzieje Zakonu Paulinów, lecz także przybliża historię eremityzmu pierwszych wieków chrześcijaństwa, z którym Paulini byli i są ściśle związani od samych początku swego istnienia. Paulina Eremus dostarcza licznych wiadomości dotyczących zarówno dziejów starochrześcijańskiego pustelnictwa, jak i samego Zakonu Paulinów. Rękopis jasno wykazuje, iż duchowość paulińskich mnichów jest głęboko związana nie tylko ze św. Pawłem z Teb - pierwszym pustelnikiem chrześcijańskim, lecz także ze wszystkimi eremitami pierwszych wieków - jego naśladowcami. 